\title{
Sequence and Structural Requirements of a Mitochondrial Protein Import Signal Defined by Saturation Cassette Mutagenesis
}

\author{
DAVID M. BEDWELL, † SCOTT A. STROBEL, KYUSON YUN, GREGG D. JONGEWARD, AND SCOTT D. EMR \\ Division of Biology, California Institute of Technology, Pasadena, California 91125
}

Received 20 October 1988/Accepted 9 December 1988

\begin{abstract}
The Saccharomyces cerevisiae $F_{1}$-ATPase $\beta$ subunit precursor contains redundant mitochondrial protein import information at its $\mathrm{NH}_{2}$ terminus (D. M. Bedwell, D. J. Klionsky, and S. D. Emr, Mol. Cell. Biol. 7:4038-4047, 1987). To define the critical sequence and structural features contained within this topogenic signal, one of the redundant regions (representing a minimal targeting sequence) was subjected to saturation cassette mutagenesis. Each of 97 different mutant oligonucleotide isolates containing single (32 isolates), double (45 isolates), or triple ( 20 isolates) point mutations was inserted in front of a $\beta$-subunit gene lacking the coding sequence for its normal import signal (codons 1 through 34 were deleted). The phenotypic and biochemical consequences of these mutations were then evaluated in a yeast strain deleted for its normal $\beta$-subunit gene ( $\Delta$ atp2). Consistent with the lack of an obvious consensus sequence for mitochondrial protein import signals, many mutations occurring throughout the minimal targeting sequence did not significantly affect its import competence. However, some mutations did result in severe import defects. In these mutants, $\beta$-subunit precursor accumulated in the cytoplasm, and the yeast cells exhibited a respiration defective phenotype. Although point mutations have previously been identified that block mitochondrial protein import in vitro, a subset of the mutations reported here represents the first single missense mutations that have been demonstrated to significantly block mitochondrial protein import in vivo. The previous lack of such mutations in the $\beta$-subunit precursor apparently relates to the presence of redundant import information in this import signal. Together, our mutants define a set of constraints that appear to be critical for normal activity of this (and possibly other) import signals. These include the following: (i) mutant signals that exhibit a hydrophobic moment greater than $\mathbf{5 . 5}$ for the predicted amphiphilic alpha-helical conformation of this sequence direct near normal levels of $\beta$-subunit import (ii) at least two basic residues are necesasry for efficient signal function, (iii) acidic amino acids actively interfere with import competence, and (iv) helix-destabilizing residues also interfere with signal function. These experimental observations provide support for mitochondrial protein import models in which both the structure and charge of the import signal play a critical role in directing mitochondrial protein targeting and import.
\end{abstract}

Greater than $90 \%$ of the proteins found in mitochondria are encoded by the nuclear genome, synthesized on cytoplasmic ribosomes, and imported into mitochondria in a coor posttranslational manner (6). These proteins usually are synthesized as larger precursors containing transient $\mathrm{NH}_{2^{-}}$ terminal presequences that are proteolytically removed upon entry into the mitochondrial matrix compartment $(11,23$, 33). It has previously been shown that information both necessary and sufficient for the import of a number of mitochondrial proteins is carried within the presequence (19, $22,35)$. These targeting signals are characteristically rich in both regularly spaced basic and hydroxyl-containing (serine and threonine) residues, they contain a number of nonpolar residues interspersed between the basic amino acids, and they are almost always devoid of acidic residues. A statistical study examining the $\mathrm{NH}_{2}$-terminal sequences of 23 mitochondrial precursor proteins found no primary amino acid sequence similarities that might indicate the existence of a consensus sequence for mitochondrial protein import signals (38). Instead, it was proposed that these targeting signals may function by virtue of their propensity to adopt an amphiphilic alpha-helical conformation that might facilitate both the initial interactions between the precursor protein and the mitochondrial surface and the subsequent translocation of the protein into the organelle. Supporting this model

$\dagger$ Present address: Department of Microbiology, University of Alabama at Birmingham, Birmingham, AL 35294. are physical studies with synthetic presequence peptides, which showed that many presequences are capable of assuming an alpha-helical configuration that can insert directly into a hydrophobic surface (such as a membrane) $(10,30$, $31)$. In addition, this insertion was stimulated when a membrane potential having the same orientation as that normally found across the mitochondrial inner membrane (electronegative inside) was introduced.

The targeting signals of a number of mitochondrial precursor proteins have been subjected to deletion analysis $(9,17$, $19,22)$. In general, most of these studies have concluded that a specific portion of the presequence is sufficient to facilitate the import of either the cognate mitochondrial protein or an unrelated passenger protein into mitochondria. However, studies analyzing point mutations, which could provide a much more detailed characterization of the sequence and structural requirements of an intact mitochondrial proteintargeting signal, have been less common. In one comprehensive in vitro study, the mitochondrial targeting signal of ornithine transcarbamylase was analyzed by using a point mutagenesis approach (16-18). These studies concluded, however, that the portion of the ornithine transcarbamylase presequence critical for mitochondrial import probably does not function as an amphiphilic alpha helix. In another study, Douglas and co-workers utilized a mutant form of the $F_{1}$-ATPase $\beta$ subunit that lacked the entire mitochondrial targeting signal to select spontaneous mutations that partially restored mitochondrial import (36). Using this ap- 
proach, they showed that mutations replacing acidic residues near the amino terminus of the deletion protein with neutral or basic residues could restore some level of import, suggesting that a less acidic amphiphilic helix was necessary to generate these surrogate mitochondrial import signals.

In another approach, random Escherichia coli DNA fragments were tested for the ability to encode a functional mitochondrial protein targeting signal (1). This was done by fusing random fragments to the gene encoding subunit IV of the cytochrome oxidase complex (missing the portion of the gene that normally encodes the targeting signal). A relatively large number of DNA inserts encoding different amino acid sequences could direct various amounts (often quite low) of the cytochrome oxidase subunit IV protein into mitochondria. These results are consistent with the idea that structural features, rather than a specific amino acid sequence, provide important functional characteristics to mitochondrial protein import signals. However, a limitation of the random-sequence approach is the difficulty in testing whether certain amino acid positions within a given import signal are particularly critical for signal function or, conversely, whether large segments of the import signal contribute collectively to the functionality of the signal. In addition, because the substituted signals vary not only in sequence composition but also in length, it is difficult to determine which portion of the substituted sequence actually contains the functional import information. Such information can best be obtained by a systematic mutational analysis of an individual targeting signal.

Recently, we found that three nonoverlapping amino acid segments near the $\mathrm{NH}_{2}$ terminus of the $\mathrm{F}_{1}$-ATPase $\beta$ subunit precursor are independently capable of directing the import of this protein into mitochondria, indicating that this mitochondrial protein import signal (and possibly others) contains redundant targeting information (2). During the course of this study, glutamic acid residues were substituted for arginine residues within the $\beta$-subunit presequence at amino acid positions 5 and 12 individually or in combination as a double mutant. Each of the resulting mutant precursor proteins was still capable of import into mitochondria in vivo at a rate sufficient to maintain wild-type levels of the protein in the organelle. Since acidic residues are thought to be detrimental to the function of mitochondrial protein import signals (38), we reasoned that the informational redundancy associated with this targeting signal acts to phenotypically suppress the adverse effects of the acidic residues. To test this model, we have subjected one of the three redundant regions previously defined in the $F_{1}$-ATPase $\beta$-subunit targeting signal (representing a "minimal targeting signal") to saturation cassette mutagenesis. Using this approach, we have isolated a large number of mutants (both functional and nonfunctional) that have allowed us to determine both structural and sequence constraints critical for the function of this targeting signal in vivo.

\section{MATERIALS AND METHODS}

Strains and media. The Saccharomyces cerevisiae strain used in this study was SEY6215 (MATa ura3-52 leu2-3,112 trp- $\Delta 901$ lys2-801 suc2- $\triangle 9$ GAL $\triangle a t p 2:: L E U 2)$. Construction of the ATP2 deletion-disruption ( $\triangle a t p 2:: L E U 2)$ was described previously (35); it was introduced into SEY6215 by using standard yeast genetic techniques (34). Control experiments verified that this strain does not produce any antigen that can be recognized by $\beta$ subunit-specific antisera. $E$. coli strains used for plasmid maintenance and M13 growth were

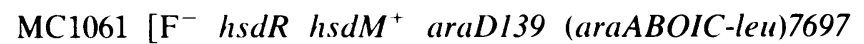
SlacX74 galU galK rpsL] (4) and JM101 [D(lac-pro) supE thi $\mathrm{F}^{\prime}$ traD36 lacIZAMI5 proAB] (23). Standard $S$. cerevisiae (34) and $E$. coli (25) media were used, and tryptophan and lysine were added as needed.

Reagents. Zymolyase-100T (Kirin Brewery Co.) was obtained from Seikagako Kogyo Co. Restriction enzymes and DNA Polymerase (Klenow fragment) were obtained from New England BioLabs, Inc., and Bethesda Research Laboratories, Inc. Proteinase $\mathrm{K}$ and $\alpha_{2}$-macroglobulin were purchased from Boehringer Mannheim Biochemicals. ${ }^{35} \mathrm{~S}$-labeled protein A was purchased from Amersham Corp., and $\left[{ }^{35} \mathrm{~S}\right]$ methionine (Tran ${ }^{35} \mathrm{~S}$-label) was purchased from ICN Pharmaceuticals Inc. Other chemicals were from Sigma Chemical Co.

Cassette oligonucleotide mutagenesis. The oligonucleotide cassette design was similar to the approach described by Hill et al. (15). A mixed oligomer 76 nucleotides long was synthesized at the Caltech Microchemical Facility with an Applied Biosystems model 380A DNA synthesizer. During synthesis, all four nucleotide precursors were added to a subset of the nucleotide coupling reactions to generate a mixed set of degenerate oligonucleotides. The mutagenesis procedure used a mixture of nucleotides at one of the first two nucleotide positions of codons 2 through 14 (the initiating methionine codon was not mutagenized). This scheme generated three potential nucleotide changes per codon, giving a total of 39 distinct amino acid substitutions obtainable in residues 2 through 14 of the minimal targeting sequence. The mutagenesis was limited to three possible amino acid changes per residue, so most of the total set of single missense mutations could be obtained. This was done to aid in the analysis of the double missense mutants, since in this way the phenotypic effect of each amino acid substitution in the double mutant could be determined independently. This greatly facilitated the interpretation of the relative contributions of each mutation within the double mutant. We biased our choice of mutations toward those that introduced as wide a range of structural and charge characteristics as possible within the limits of the genetic code. In three cases (codons 3,8 , and 11) the natural codon usage was changed to an alternative codon (while conserving the wildtype amino acid encoded at that position) to allow a broader range of informative amino acid substitutions.

The ratio of mixed nucleotides used was 33 (wild type): $1: 1: 1$ (mutant), which was calculated to provide the optimal probability of one mutation per oligonucleotide molecule. The resulting oligonucleotides encoded 22 bases $5^{\prime}$ of the ATG start codon as well as the first 14 codons of the $F_{1}$-ATPase $\beta$-subunit presequence and was designed to include flanking HindIII and SalI sites (Fig. 1). A complementary 11-nucleotide oligomer was annealed to the $3^{\prime}$ end of the 76-mers, and duplex DNA molecules were generated by chain polymerization with the Klenow fragment of DNA polymerase $\mathrm{I}$. This resulted in molecules containing the nucleotide substitutions fixed into both strands of the resulting duplex molecules. The double-stranded molecules were cleaved with HindIII and SalI and then ligated into the HindIII and SalI sites of a CEN ARS plasmid containing a truncated $A T P 2$ gene lacking the coding sequence for residues 1 through 34 of the wild-type $\beta$-subunit precursor and 22 nucleotides $5^{\prime}$ of the ATG start codon (Fig. 1). The resulting ligated molecules encoded a $\beta$-subunit precursor containing residues 1 through 14 and 35 through 510 (residues 15 through 34 were deleted). The ligation mix was transformed into $E$. coli $\mathrm{MC1061}$, and independent trans- 

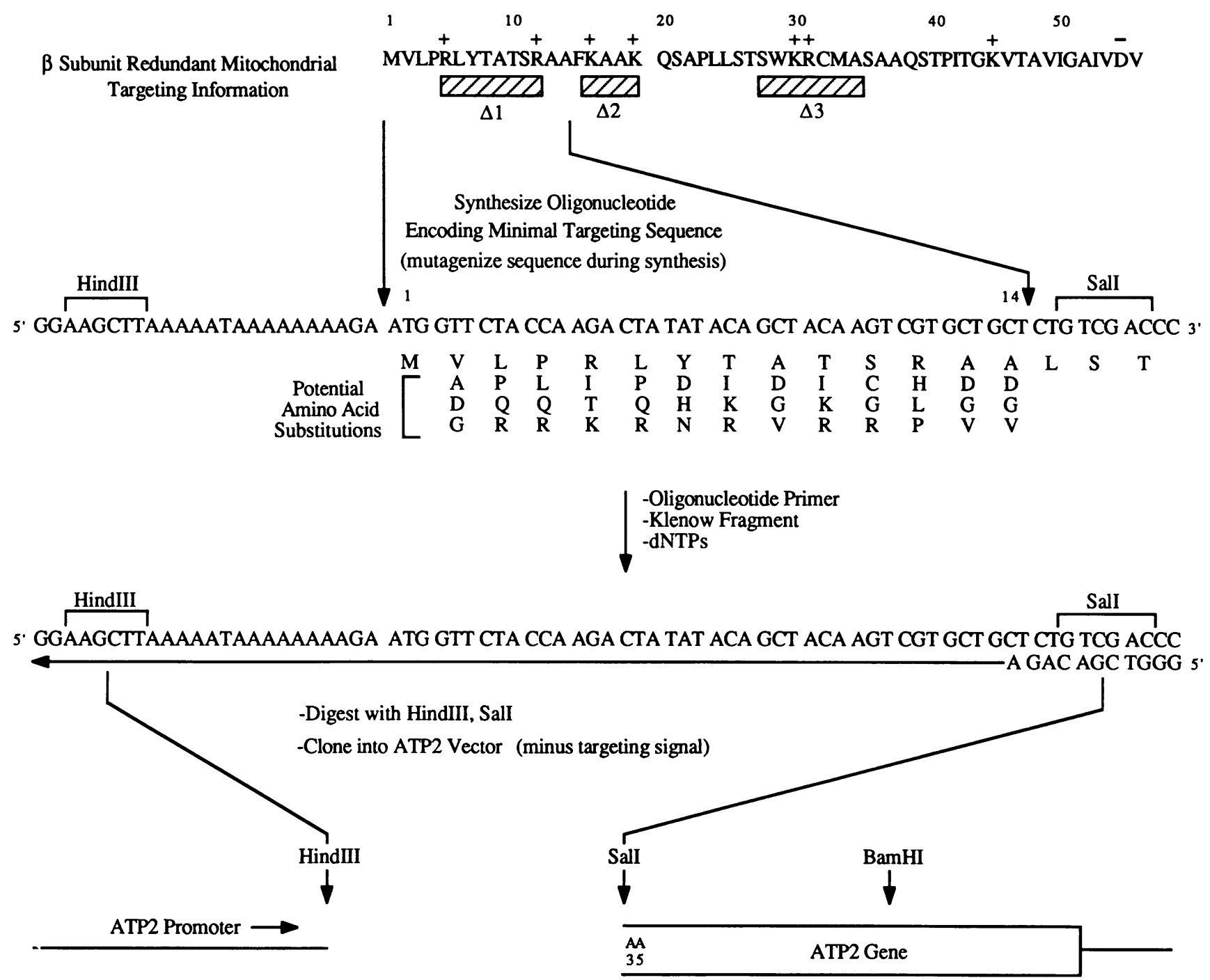

FIG. 1. Outline of oligonucleotide cassette mutagenesis approach. The $\mathrm{NH}_{2}$ terminus of the $\beta$-subunit precursor is shown in the first line, with cross-hatched boxes representing deletions of regions containing functionally redundant targeting information that were described previously (2). The second line shows the 76-base mixed oligonucleotide, with both the wild-type amino acid sequence and potential amino acid substitutions encoded by the oligomer indicated with the single-letter code. The remainder of the figure shows sequentially the steps involved in the mutagenesis procedure as outlined in the Materials and Methods. The resulting plasmid constructs encode the 14-amino-acid minimal targeting sequence and the 3 amino acids encoded by the Sall site, followed immediately by the serine residue at position 35 of the mature $\beta$-subunit sequence.

formants were isolated. Plasmid DNA was recovered from individual transformants, and a HindIII-BamHI fragment from each was subcloned into M13mp8. Single-stranded DNA was then analyzed by dideoxy DNA sequencing (32). Plasmids containing mutations of interest were then transformed into SEY6215 by the lithium acetate method (21). Because this strain carries a deletion-disruption of the ATP2 locus, the ability of each mutant $\beta$-subunit precursor to restore growth on a nonfermentable carbon source could then be tested.

Subcellular fractionation and immunoblots. Yeast' cells carrying the indicated plasmids were grown at $28^{\circ} \mathrm{C}$ in yeast nitrogen base medium (YNB; Difco Laboratories) containing D-galactose as the carbon source to an $A_{600}$ of 0.5 to 1.0 . Both spheroplasting and subcellular fractionation were done as described previously $(2,5)$, with the following modifications. We had previously experienced difficulty in stabilizing at least one unassembled mutant form of the $\beta$-subunit protein during subcellular fractionation procedures following cell lysis (2). To circumvent this problem, a cocktail of protease inhibitors was included during cell breakage and in each of the subsequent fractionation steps. This cocktail included $1 \mathrm{mM}$ phenylmethylsulfonyl fluoride, $0.1 \mathrm{mM} \mathrm{N}$ $\alpha$-p-tosyl-L-lysine chloromethyl ketone, and $100 \mu \mathrm{g}$ of $\alpha_{2-}$ macroglobulin per $\mathrm{ml}$. This modification resulted in a more quantitative recovery of the mutant proteins regardless of their subcellular location, as judged by normalization of the recovery of the total amount (cytosolic and mitochondrial) of the $F_{1}-\beta$ subunit to various marker proteins. Efficient recovery of the $\beta$ subunit was also indicated by experiments that compared the total amount of this protein recovered from equivalent numbers of cells from mutants either able to grow on lactate media $\left(\mathrm{Lct}^{+}\right)$or unable to grow on lactate media $\left(\mathrm{Lct}^{-}\right)$. This allowed us to compare the relative amount of the mutant $\beta$-subunit proteins targeted to mitochondria in each mutant. The efficiency of fractionation and recovery 
was monitored by assaying fractions for the cytosolic marker $\alpha$-glucosidase (13) and by probing immunoblots for the mitochondrial outer membrane porin protein. In all cases, $>90 \%$ of each of these markers was localized to the appropriate subcellular fraction. Published procedures were used to assay protein concentration (3).

For immunoblot analysis of subcellular fractions, cellequivalent amounts of cytosolic and mitochondrial protein (100 $\mu \mathrm{g}$ of cytosolic protein and approximately $10 \mu \mathrm{g}$ of mitochondrial protein) were electrophoresed in adjacent lanes of a $10 \%$ polyacrylamide-SDS gel. Proteins were transferred to a GeneScreen membrane (Dupont, NEN Research Products) under the conditions recommended by the manufacturer. The primary antibodies used in immunoblotting were monoclonal antibodies to the $F_{1}$-ATPase $\beta$ subunit and polyclonal antisera to the mitochondrial outer membrane porin (both gifts from G. Schatz) by using a procedure provided by DuPont, NEN. The membrane was washed and then incubated with rabbit anti-mouse serum. The membrane was again washed, and antibodies bound to specific antigens were detected by incubating the membrane with ${ }^{35} \mathrm{~S}$-labeled protein $\mathrm{A}$, followed by autoradiography. Quantitation of autoradiographs was done with an LKB densitometer.

Protease treatment of mitochondria. Mitochondria $(75 \mu \mathrm{g})$ were incubated for $30 \mathrm{~min}$ on ice with $50 \mu \mathrm{g}$ of proteinase $\mathrm{K}$ per $\mathrm{ml}$. Bovine serum albumin $(3 \mathrm{mg} / \mathrm{ml})$ was also included in each reaction. For membrane permeabilization, $0.5 \%$ Triton $\mathrm{X}-100$ was included. The reaction was terminated by the addition of $1 \mathrm{mM}$ phenylmethylsulfonyl fluoride. Mitochondria were then isolated by centrifugation through a cushion containing $0.6 \mathrm{M}$ sucrose, $10 \mathrm{mM}$ MOPS (morpholinepropanesulfonic acid) ( $\mathrm{pH} \mathrm{7.2),} \mathrm{and} 1 \mathrm{mM}$ EDTA.

Radiolabeling and immunoprecipitations. Cultures were grown to an $A_{600}$ of 0.5 to 1.0 in YNB medium containing D-galactose as the carbon source. Cells were harvested and suspended in the same medium at a concentration of 2 to 4 $A_{600}$ units per ml. The pulse period was initiated by the addition of $250 \mu \mathrm{Ci}$ of $\left[{ }^{35} \mathrm{~S}\right]$ methionine per $\mathrm{ml}$. The chase period was started 4 min later by the addition of both $50 \mu \mathrm{g}$ of unlabeled methionine per $\mathrm{ml}$ and $100 \mu \mathrm{g}$ of cycloheximide per $\mathrm{ml}$, and $0.5-\mathrm{ml}$ samples were harvested at the indicated times by pipetting directly into trichloroacetic acid ( $5 \%$, final concentration). Immunoprecipitations were carried out as previously described (2).

\section{RESULTS}

Mutagenesis strategy and distribution of mutations. We previously found that the mitochondrial protein import signal of the $F_{1}$-ATPase $\beta$-subunit precursor contains functionally redundant targeting information (2). To identify the important features defining one of these elements, we subjected a single redundant element of the $F_{1}$-ATPase $\beta$ subunit targeting signal to saturation cassette mutagenesis. A mixed population of wild-type and mutant oligonucleotides encoding the minimal $\beta$-subunit targeting sequence was ligated to a version of the ATP2 gene (lacking its mitochondrial protein targeting information; see Materials and Methods) on the low-copy-number CEN4 ARS1 shuttle vector pSEYC58 (9) (Fig. 1). The ligation mixture was then used to transform $E$. coli, and plasmid constructs isolated from individual colonies were sequenced. Of the 213 sequenced clones, 97 unique mutants were obtained that uniformly saturated the 13-amino-acid target region with 32 single, 45 double, and 20 triple missense mutants (Table 1). Other
TABLE 1. Distribution of mutations ${ }^{a}$

\begin{tabular}{llcc}
\hline $\begin{array}{c}\text { Position } \\
\text { (amino } \\
\text { acid) }\end{array}$ & \multicolumn{2}{c}{ No. of mutants with the following mutations: } \\
\cline { 2 - 4 } & Single & Double & Triple \\
\hline 1 (Met) & & & \\
2 (Val) & 3 & 6 & 3 \\
3 (Leu) & 1 & 7 & 8 \\
4 (Pro) & 3 & 8 & 6 \\
5 (Arg) & 3 & 7 & 4 \\
6 (Leu) & 3 & 3 & 2 \\
7 (Tyr) & 2 & 10 & 7 \\
8 (Thr) & 3 & 8 & 4 \\
9 (Ala) & 2 & 5 & 5 \\
10 (Thr) & 3 & 7 & 5 \\
11 (Ser) & 3 & 8 & 4 \\
12 (Arg) & 2 & 5 & 4 \\
13 (Ala) & 2 & 8 & 6 \\
14 (Ala) & 2 & 8 & 2 \\
\hline
\end{tabular}

a Total isolates included 80 wild types, 67 single mutants, 46 double mutants, and 20 triple mutants. Unique isolates included 1 wild type, 32 single mutants, 45 double mutants, and 20 triple mutants. The percentages of mutants observed and percentages expected, respectively, were as follows: 39 and $35 \%$ wild types, 29 and $38 \%$ single mutants, 20 and $19 \%$ double mutants. and 9 and $6 \%$ triple mutants.

plasmid isolates encoded either the wild-type sequence for residues 1 through 14 [called WT(1-14)], duplicate single and double mutant forms of the sequence, or quadruple and quintuple mutants that were not analyzed further. Plasmids encoding mutant isolates were designated by the single-letter code for the wild-type amino acid followed by the position number and the single-letter code for the mutant residue (e.g., a plasmid encoding a mutation that changed the arginine residue at position 5 to lysine was called R5-K).

Mitochondrial localization and processing of mutant $\beta$ proteins. Mutant yeast strains unable to target the $\beta$ subunit to mitochondria and assemble a functional mitochondrial proton-translocating ATPase complex cannot grow on a nonfermentable carbon source such as lactate (2). To test the functional competence of the mutant targeting signals, plasmids carrying each of the mutant $A T P 2$ genes were transformed into the yeast strain SEY6215 ( $\Delta a t p 2)$, and the growth phenotypes of the resulting transformants were determined on YP lactate plates. Three general growth phenotypes were found: wild-type growth at 30 and $37^{\circ} \mathrm{C}\left(\mathrm{Lct}^{+}\right)$, growth at $30^{\circ} \mathrm{C}$ but not at $37^{\circ} \mathrm{C}[\mathrm{Lct}(\mathrm{Ts})]$, and no growth on lactate $\left(\mathrm{Lct}^{-}\right)$(Fig. 2). Of the 32 unique single missense mutants isolated, 14 displayed either a partially or completely defective growth phenotype [either Lct(Ts) or $\mathrm{Lct}^{-}$]. The growth characteristics of the other 18 isolates were phenotypically indistinquishable from that obtained with either the wild-type ATP2 gene or the WT(1-14) construct on YP-lactate medium.

Because phenotypic complementation does not in itself provide a quantitative measure of the amount of mutant $\beta$-subunit precursor that has been imported into mitochondria, we fractionated yeast cells into mitochondrial and cytosolic cell fractions and assayed the amount of $\beta$ subunit in each fraction. Cell-equivalent amounts of each fraction were then subjected to immunoblot analysis (Fig. 3). By comparing the amount of $\beta$-subunit protein in each fraction with the total amount of $\beta$ subunit, the steady-state distribution could be determined for each mutant protein. A very strong correlation was seen between the level of $\beta$-subunit accumulation in mitochondria and the growth phenotypes exhibited by each mutant on lactate media (Table 2). All of 


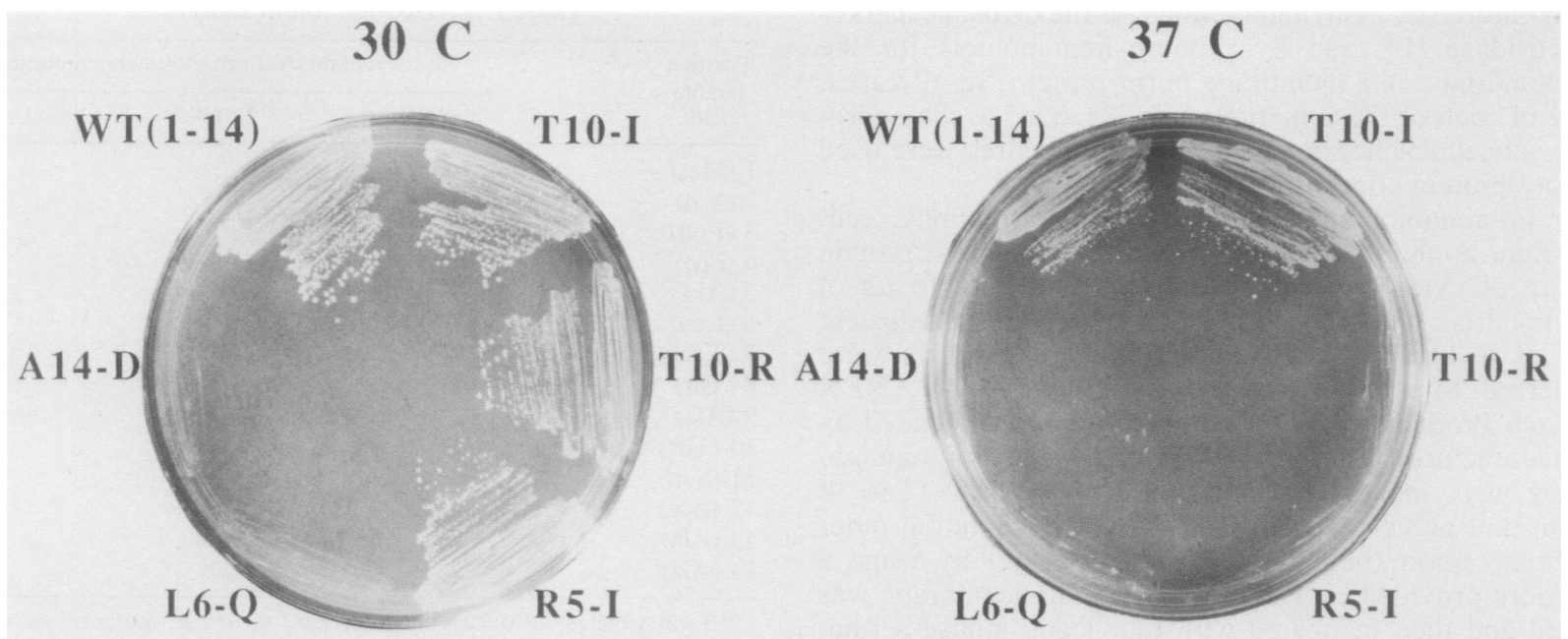

FIG. 2. Growth of representative $\beta$-subunit missense mutants on YP-lactate medium at $30^{\circ} \mathrm{C}$ and $37^{\circ} \mathrm{C}$. Selected mutants show examples of the $\mathrm{Lct}^{+}, \mathrm{Lct}^{t s}$, and $\mathrm{Lct}^{-}$growth phenotypes. Mutants are designated by the single-letter code for the wild-type amino acid followed by the position number and the single-letter code for the mutant residue.

the $\mathrm{Lct}^{+}$mutants targeted 80 to $100 \%$ of the $\beta$ subunit to mitochondria; all of the Lct(Ts) mutants had 30 to $60 \%$ of the $\beta$ subunit in mitochondria; and all of the Lct $^{-}$mutants localized $25 \%$ or less of the $\beta$ subunit to mitochondria. These results demonstrate that the growth phenotype on this medium is a function of the level of mitochondrial localization of the mutant $\beta$-subunit protein, and the amount of these mutant $\beta$ subunit proteins targeted to mitochondria can be predicted (within limits) solely by their growth phenotype on YP-lactate medium.

Of the total single missense mutants that were isolated, $25 \%$ (8 of 32 ) had a Lct(Ts) phenotype. This result was

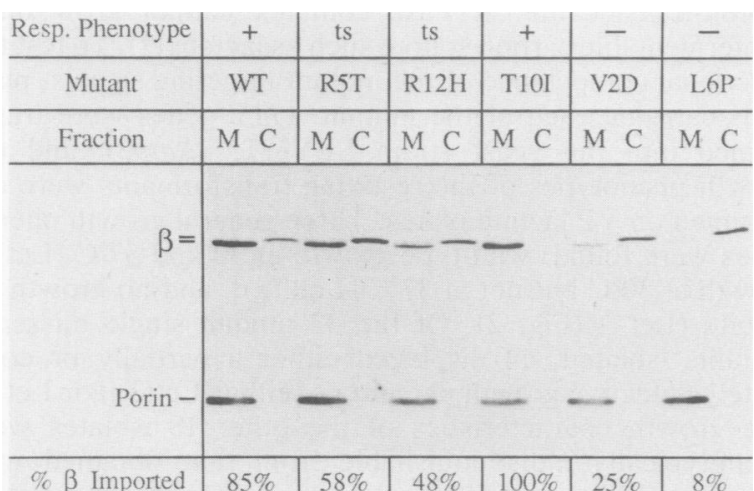

FIG. 3. Immunoblot showing the intracellular location of selected $\beta$-subunit mutant proteins. Cultures of SEY6215 expressing either the WT(1-14) $\beta$-subunit construct or a mutant form of the construct were grown at $28^{\circ} \mathrm{C}$ in YNB medium containing $2 \%$ galactose as the carbon source. After harvesting, cells were treated with the enzyme preparation Zymolyase-100T to form spheroplasts. They were then disrupted in the presence of protease inhibitors and fractionated into mitochondrial (M) and cytosolic $(C)$ fractions, and cell-equivalent amounts of each fraction were resolved by sodiun dodecyl sulfate-polyacrylamide gel electrophoresis and immunoblotted. Antibodies to the mitochondrial outer membrane porin were included only as a fractionation control, since the signal for this protein (unlike the signal for the $\beta$ subunit) was rather variable. Also shown is a quantitation of the amount of $F_{1}$-ATPase $\beta$ subunit antigen detected in the mitochondrial fraction relative to the total amount of antigen found in both fractions (\% $\beta$ Imported).
TABLE 2. Characterization of single mutants

\begin{tabular}{|c|c|c|c|c|c|}
\hline Mutant & $\begin{array}{c}\text { Lactate } \\
\text { phenotype }\end{array}$ & $\begin{array}{c}\% \\
\text { Imported }\end{array}$ & Range $^{a}$ & $\begin{array}{l}\text { Hydrophobic } \\
\text { moment }^{b}\end{array}$ & $\begin{array}{l}\text { Chou- } \\
\text { Fasman } \\
\text { change }\end{array}$ \\
\hline True wild type & + & 100 & $3-20$ & 10 & \\
\hline WT (1-14) & + & $85-90$ & $3-20$ & 5.8 & \\
\hline V2-A & + & $90-100$ & $2-19$ & 5.8 & h-H \\
\hline V2-L & + & $90-100$ & $3-20$ & 5.8 & h-H \\
\hline L3-R & + & $90-100$ & $5-22$ & 4 & $\mathrm{H}-\mathrm{i}$ \\
\hline P4-R & + & $90-100$ & 2-19 & 7.1 & $B-i$ \\
\hline P4-Q & + & $90-100$ & $2-19$ & 6.2 & B-h \\
\hline P4-L & + & $90-100$ & $3-20$ & 5.7 & B-H \\
\hline R5-K & + & $90-100$ & $2-19$ & 4.9 & $i-h$ \\
\hline T8-I & + & $90-100$ & $3-20$ & 4.7 & $i-h$ \\
\hline T10-I & + & $90-100$ & $3-20$ & 7.2 & $i-h$ \\
\hline S11-R & + & $90-100$ & $2-19$ & 6.2 & $i-i$ \\
\hline S11-C & + & $90-100$ & $3-20$ & 5.9 & $\mathrm{i}-\mathrm{i}$ \\
\hline S11-G & + & $90-100$ & $3-20$ & 5.9 & $\mathrm{i}-\mathrm{B}$ \\
\hline A13-V & + & $90-100$ & $2-19$ & 6 & H-h \\
\hline A14-V & + & $90-100$ & $3-20$ & 6.2 & H-h \\
\hline T8-R & + & $80-90$ & $2-19$ & 8 & $i-i$ \\
\hline T8-K & + & $80-90$ & 2-19 & 7.1 & i-h \\
\hline A9-V & + & $80-90$ & $2-19$ & 5.6 & H-h \\
\hline A13-G & + & $80-90$ & $3-20$ & 5.8 & H-B \\
\hline R5-T & Ts & $45-60$ & $2-19$ & 3.8 & $i-i$ \\
\hline R5-I & Ts & $45-60$ & 2-19 & 3 & $\mathrm{i}-\mathrm{h}$ \\
\hline Y7-H & Ts & $45-60$ & $2-19$ & 5.4 & b-I \\
\hline T10-R & Ts & $45-60$ & $3-20$ & 3.5 & $\mathrm{i}-\mathrm{i}$ \\
\hline R12-H & Ts & $45-60$ & $3-20$ & 3.7 & $\mathrm{i}-\mathrm{I}$ \\
\hline Y7-N & Ts & $30-45$ & $2-19$ & 5.2 & b-b \\
\hline A9-G & Ts & $30-45$ & $3-20$ & 5.9 & H-B \\
\hline T10-K & Ts & $30-45$ & $3-20$ & 4.4 & $\mathrm{i}-\mathrm{h}$ \\
\hline V2-D & - & $15-25$ & $2-19$ & 6.2 & h-H \\
\hline L6-R & - & $15-25$ & $3-20$ & 4.9 & $\mathrm{H}-\mathrm{i}$ \\
\hline R12-P & - & $15-25$ & $2-19$ & 3.2 & i-B \\
\hline L6-P & - & $0-10$ & $3-20$ & 5.4 & H-B \\
\hline L6-Q & - & $0-10$ & $3-20$ & 5 & H-h \\
\hline A14-D & - & $0-10$ & $3-20$ & 6.2 & H-I \\
\hline
\end{tabular}

a Range indicates the specific 18 amino acid residues that yield the maximal hydrophobic moment value.

${ }_{b}$ Calculated using the equation of Eisenberg $(7,8)$ with a scanning window of 18 residues. 


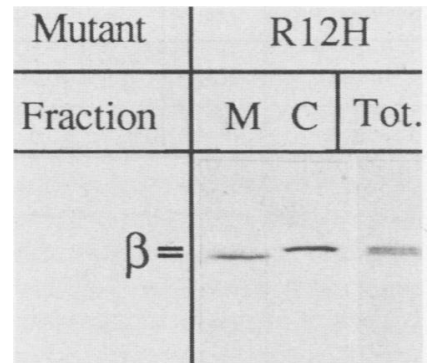

FIG. 4. Immunoblot comparing fractionated and total cellular samples of a representative $\beta$-subunit missense mutant protein. The total cellular sample (Tot) was prepared as a trichloroacetic acid precipitate of an exponentially growing culture as described for the immunoprecipitation procedure in Materials and Methods. Fractionated samples were prepared as described in the legend to Fig. 3 and in Materials and Methods.

surprising, because it previously has been extremely difficult to isolate conditional mutations in other topogenic signals. This phenotype could result from a temperature-sensitive function of the signal or as a secondary consequence of some other defect, such as instability of the mutant precursor protein at the restrictive temperature. Immunoblots of fractionated samples from each of the $\mathrm{Lct}^{t s}$ single missense mutants showed that the ratio of $\beta$ subunit detectable in the two subcellular fractions was similar when cultures were grown at 28 and $37^{\circ} \mathrm{C}$. However, the total amount of the mutant precursors [relative to the WT(1-14) control] was decreased severalfold at the restrictive temperature, indicating that degradation was occurring (data not shown). This suggests that the temperature-sensitive phenotype of these mutant precursors is a direct consequence of precursor degradation. This degradation apparently results in the mitochondrial localization of an amount of $\beta$-subunit protein that is insufficient to support growth on lactate media at the restrictive temperature.

A difference in mobility between the cytosolic and mitochondrial $\beta$ subunit species was also observed in many cases (Fig. 3). To further analyze the cause of this apparent difference, whole cell samples were harvested from selected mutants by trichloroacetic acid precipitation and subjected directly to immunoblot analysis (Fig. 4). Two bands were clearly visible in these samples, suggesting that some form of proteolytic processing might be occurring upon import. This was confirmed by pulse-chase experiments in which it was shown that processing was blocked by either the uncoupling agent carbonyl cyanide $m$-chlorophenylhydrozone, a compound that rapidly blocks mitochondrial protein import by eliminating the electrochemical potential across the inner mitochondrial membrane (28); or by 1,10 -phenanthroline, a membrane-permeable chelator that inhibits the matrix processing protease (data not shown) (14). These results provide strong evidence that these mutant $\beta$-subunit precursors are processed by the matrix processing protease upon import into the mitochondria. The cleavage site in these mutant precursors has not yet been determined, but the previously assigned processing site is not present (35).

Although in no case was mature $\beta$-subunit protein found in the cytosolic fraction, some mutant constructs could be shown to contain the precursor form of the $\beta$ subunit in the mitochondrial fraction. To determine whether this represented mutants partially defective for recognition by the matrix-processing protease or precursor that was bound to the surface of mitochondria but not yet imported, isolated

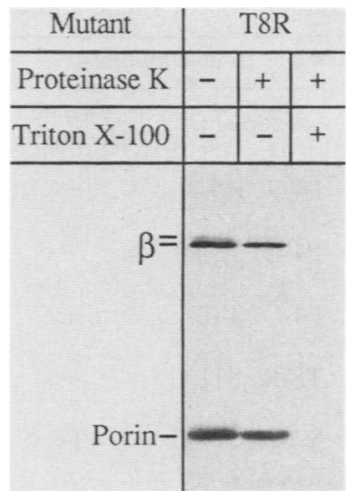

FIG. 5. Immunoblot of a representative mutant $\beta$-subunit protein that accumulates $\beta$-subunit precursor in the mitochondrial fraction after protease treatment of isolated mitochondria.

mitochondria from representative mutants were subjected to a limited proteolytic treatment with proteinase K (Fig. 5). Much of the precursor in each of three different mutants was resistant to the protease unless detergent was also added to disrupt the mitochondrial membranes. This indicates that at least some of the precursor protein must be located within the mitochondria and that these mutations partially disrupt either the matrix protease recognition site, the cleavage site, or both. Interestingly, many of the muant $\beta$-subunit precursors that accumulated in the mitochondrial fraction contained amino acid substitutions that either added or removed a charged residue, suggesting that the spatial presentation of charged residues may be important for proper recognition or cleavage by the matrix protease.

Some targeting signal mutations increase the rate of import. Fractionation experiments indicated that some mutant targeting signals accumulate a larger fraction of the total $\beta$ subunit within mitochondria $(100 \%)$ than the isogenic WT(114) construct (85\%; Fig. 3). These results suggest that these mutants may also be imported at a faster rate. To test this possibility, import kinetics (as measured by the rate of processing) were determined for the WT(1-14) parent and two mutants that accumulated increased steady-state amounts of the mature $\beta$-subunit protein in the mitochondrial fraction (Fig. 6). After a 4-min pulse with $\left[{ }^{35} \mathrm{~S}\right] \mathrm{methio}-$ nine followed by a 10 -min chase, only $50 \%$ of the WT(1-14) construct was processed to the mature form. In contrast, both mutants tested showed $50 \%$ or more precursor processed to the mature form after only a $2.5-\mathrm{min}$ chase, a

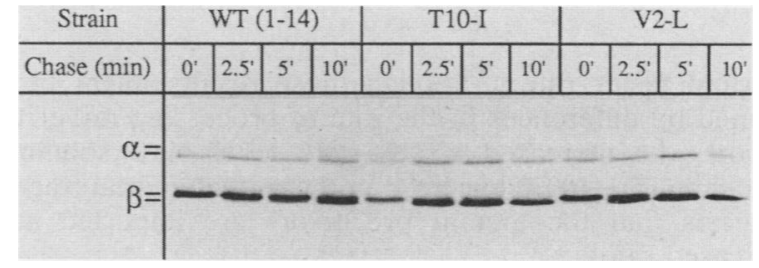

FIG. 6. Processing kinetics of selected $\beta$-subunit mutant precursors. SEY6215 cells expressing either the WT(1-14) $\beta$-subunit precursor or mutant forms of this construct were labeled for $4 \mathrm{~min}$ with $\left[{ }^{35} \mathrm{~S}\right]$ methionine, followed by a chase in the presence of $50 \mu \mathrm{g}$ of unlabeled methionine per $\mathrm{ml}$ and $100 \mu \mathrm{g}$ of cycloheximide per $\mathrm{ml}$. The chase was terminated at the indicated times by the addition of trichloroacetic acid. The positions of the $\alpha$ and $\beta$ subunits of the $F_{1}$-ATPase complex are marked. 
TABLE 3. Comparison of single and double mutants

\begin{tabular}{|c|c|c|c|c|}
\hline \multirow{2}{*}{$\begin{array}{l}\text { Combined phenotype } \\
\text { class }\end{array}$} & \multicolumn{2}{|c|}{ Double mutants } & \multicolumn{2}{|c|}{ Single mutants } \\
\hline & Mutant & $\begin{array}{c}\text { Lactate } \\
\text { phenotype }\end{array}$ & Mutant & $\begin{array}{c}\text { Lactate } \\
\text { phenotype }\end{array}$ \\
\hline \multirow[t]{12}{*}{ 1. Silent } & P4-R, R5-K & + & P4-R & + \\
\hline & & & R5-K & + \\
\hline & P4-L, T8-R & + & P4-L & + \\
\hline & & & T8-R & + \\
\hline & P4-Q, T10-I & + & P4-Q & + \\
\hline & & & T10-I & + \\
\hline & T8-R, S11-C & + & T8-R & + \\
\hline & & & S11-C & + \\
\hline & Y7-H, A9-G & Ts & Y7-H & Ts \\
\hline & & & A9-G & Ts \\
\hline & Y7-H, T10-K & Ts & $\mathrm{Y} 7-\mathrm{H}$ & Ts \\
\hline & & & $\mathrm{T} 10-\mathrm{K}$ & Ts \\
\hline \multirow{5}{*}{$\begin{array}{l}\text { 2. Complete } \\
\text { suppression }\end{array}$} & T10-I, R5-I & + & T10-I & + \\
\hline & S11 $\mathrm{DF}$ & t & $\begin{array}{l}\text { KJ-1 } \\
\text { C11 }\end{array}$ & $1 \mathrm{~s}$ \\
\hline & & & R5-I & Ts \\
\hline & T8-K, A14-D & + & $\mathrm{T} 8-\mathrm{K}$ & + \\
\hline & & & A14-D & - \\
\hline \multirow{4}{*}{$\begin{array}{l}\text { 3. Partial } \\
\text { suppression }\end{array}$} & P4-R, R12-P & Ts & P4-R & + \\
\hline & & & R12-P & - \\
\hline & S11-C, L6-Q & Ts & S11-C & + \\
\hline & & & L6-Q & - \\
\hline \multirow[t]{14}{*}{ 4. Nonsuppression } & P4-L, Y7-N & Ts & P4-L & + \\
\hline & & & Y7-N & Ts \\
\hline & P4-R, Y7-N & Ts & P4-R & + \\
\hline & & & Y7-N & Ts \\
\hline & P4-R, A9-G & Ts & P4-R & + \\
\hline & & & A9-G & Ts \\
\hline & A9-G, A13-V & Ts & A13-V & + \\
\hline & & & A9-G & Ts \\
\hline & S11-C, V2-D & - & S11-C & + \\
\hline & & & V2-D & - \\
\hline & P4-R, L6-Q & - & P4-R & + \\
\hline & & & L6-Q & - \\
\hline & R5-T, A14-D & - & R5-T & Ts \\
\hline & & & A14-D & - \\
\hline \multirow[t]{10}{*}{ 5. Antagonism } & V2-A, L3-R & Ts & V2-A & + \\
\hline & & & L3-R & + \\
\hline & T8-K, A13-G & Ts & T8-K & + \\
\hline & & & A13-G & + \\
\hline & T10-I, S11-G & - & T10-I & + \\
\hline & & & S11-G & + \\
\hline & T8-R, Y7-H & - & T8-R & + \\
\hline & & & Y7-H & Ts \\
\hline & T10-R, S11-R & - & T10-R & + \\
\hline & & & S11-R & Ts \\
\hline
\end{tabular}

fourfold faster rate. Although these results might be explained by differences in the rate of processing rather than import, the increased steady-state level of $\beta$ subunit in mitochondria ( $100 \%$ localized in the mitochondrial fraction) suggests that the mutant precursors are imported at an increased rate.

Interaction between mutations within the targeting signal. As mentioned above, 45 isolates contained two separate missense mutations within the targeting signal. Of these 45 double mutants, 23 contained pairs of mutations that had been isolated separately as single mutants. Analysis of the growth characteristics of these double mutants on lactate media, combined with the lactate growth phenotypes con-

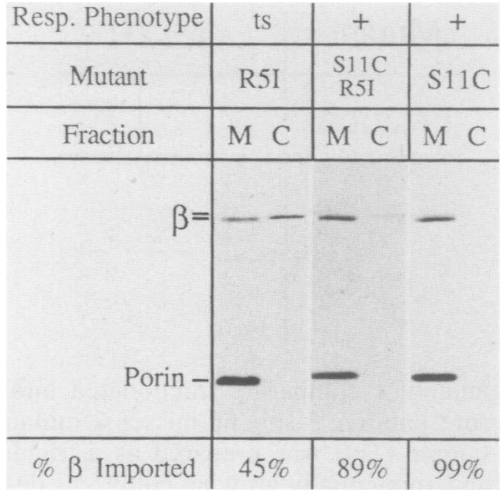

FIG. 7. Immunoblot of a class 2 double missense mutant demonstrating intragenic suppression. "\% $\beta$ Imported" is a quantitation of the amount of $\beta$-subunit antigen detected in the mitochondrial fraction relative to the total amount of antigen found in both fractions.

ferred by each mutation individually, allowed us to separate the double mutant into five distinct classes (Table 3). The first of these (the "silent" class) consists of isolates in which each double mutant has the same lactate phenotype as that of mutants carrying either mutation separately. This suggests that these mutations do not significantly interfere with import signal structure or function, either individually or in combination.

The next three classes of mutants are defined by various levels of phenotypic suppression of one mutation by the second mutation. Class 2 contains double mutants in which one mutation exercises complete suppression over the growth defect caused by the other mutation, an interaction classically termed intragenic suppression. An immunoblot of a representative of this class demonstrates that the intragenic suppression phenotype reflects a real increase in $\beta$-subunit import into mitochondria (Fig. 7). Class 3 (partial suppression) contains double mutants that demonstrate a weaker level of intragenic suppression, in which a temperature sensitive phenotype (intermediate between the phenotypes of the two single mutants) is found. Class 4 contains isolates that demonstrate an interaction we call nonsuppression, a situation where the more defective phenotype of the two single mutants is expressed in the double mutant. Each of these three classes demonstrates different interactions between the individual mutations within the targeting signal, and the data strongly suggest that the amino acids that make up the targeting signal act in a collective manner, possibly by stabilizing a secondary structure important for signal recognition.

Class 5, the "antagonism" class, represents isolates in which the double mutant grows less well on lactate medium than either of the single mutants individually. This indicates that one or both mutations function less efficiently in the environment of the double mutant than either can function separately. Interestingly, four of the five representatives of this class contain pairs of mutations that occur at adjacent amino acid residues. The antagonism class thus gives a clear indication of the stringent local constraints placed upon the residues within the targeting signal and demonstrates that a wide range of random changes cannot be tolerated in a minimal mitochondrial protein-targeting signal.

Parameters that define a functional import signal. When the single missense mutations were placed on a linear representation of the minimal targeting signal, the mutations leading 
A
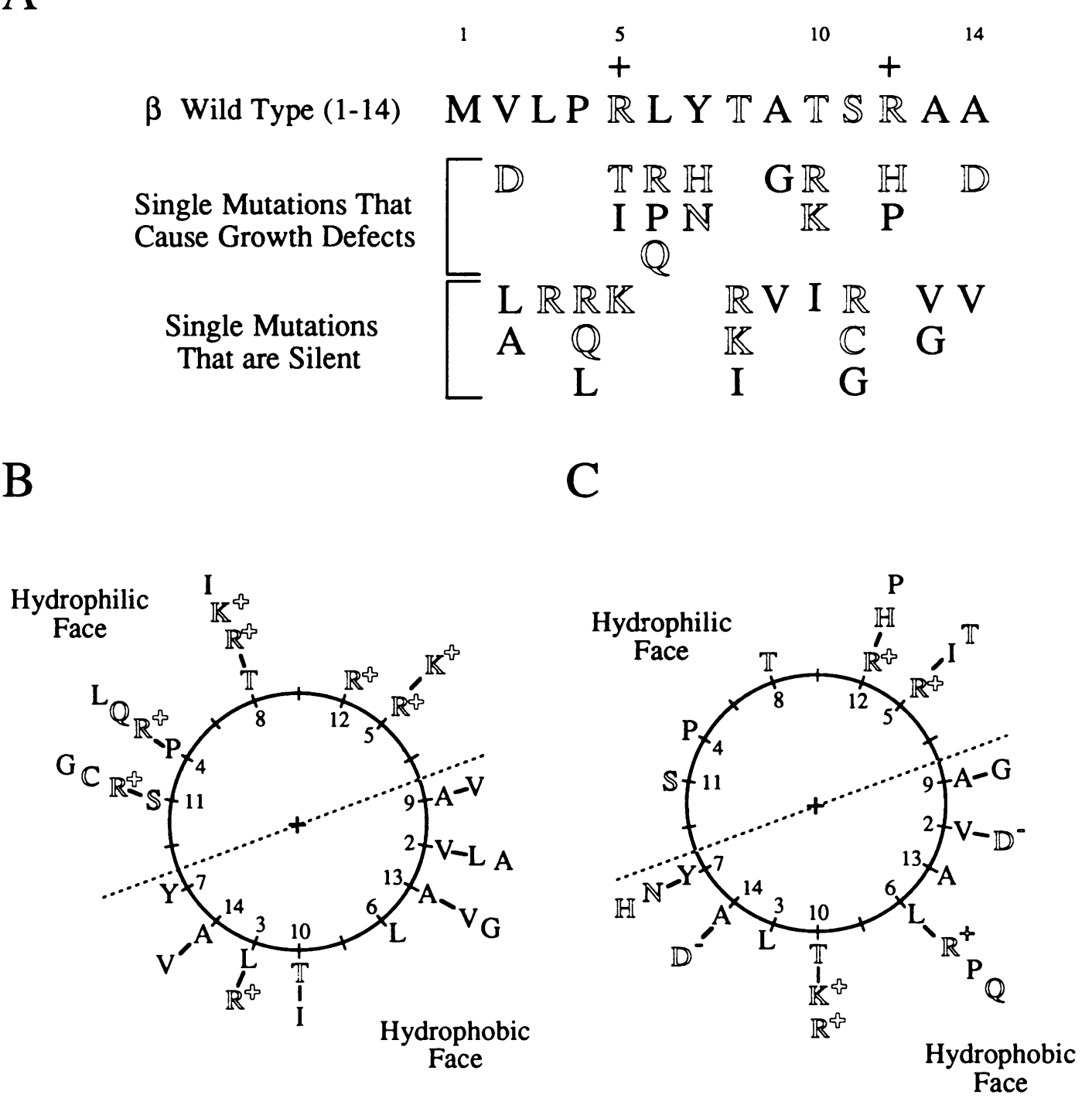

FIG. 8. Representation of phenotypically silent $\left(\mathrm{Lct}^{+}\right)$and phenotypically defective $\left(\mathrm{Lct}^{t s}\right.$ and $\left.\mathrm{Lct}^{-}\right) \beta$-subunit single missense mutations on linear and helical wheel diagrams. Hydrophobic residues are indicated by filled letters, and hydrophilic residues are indicated by open letters. In the composite helical wheel diagrams, the letters next to the circle represent the wild-type residues, whereas the more distal letters represent the various mutant residues isolated at positions 2 through 14 . (A) Linear map of phenotypically defective and phenotypically silent single missense mutations; (B) composite helical wheel diagram of phenotypically silent $\left(\mathrm{Lct}^{+}\right) \beta$-subunit single missense mutations; (C) composite helical wheel diagram of phenotypically defective $\left(\mathrm{Lct}^{t s}\right.$ and $\left.\mathrm{Lct}^{-}\right) \beta$-subunit single missense mutations.

to different growth phenotypes were interspersed throughout the sequence in a seemingly random manner (Fig. 8A). However, when the mutant residues were collectively placed on a helical wheel projection, which approximates the two-dimensional distribution of amino acid residues arrayed about the longitudinal axis of an alpha helix, clear trends became apparent. Based upon projections of both the phenotypically silent mutant signals (Fig. 8B) and mutant signals that cause lactate growth defects (Fig. 8C), the importance of the amphiphilic nature of the helix becomes apparent. Either the substitution of polar residues on the hydrophobic side of the helix or a loss of basic residues on the hydrophilic side of the helix results in a lactate growth defect. However, conservative amino acid changes on the hydrophobic surface and a wide range of changes on the hydrophilic surface at positions other than the basic residues are silent. This indicates that the basic residues may be the only critical hydrophilic residues and that the other residues on the hydrophilic surface may be constrained only in that they must promote (or at least not disrupt) the alpha-helical structure of the signal. Overall, these changes are as predicted if the targeting signal functions in an amphiphilic alpha-helical conformation.

Several mutant signals containing only one of the two basic residues that occur in the WT(1-14) signal were still functional, although at a reduced level (45 to $60 \%$ imported; see mutants R5-T, R5-I, and R12-H in Table 2). This demonstrates that two basic residues are required for efficient import but only one basic residue is necessary for a partially functional mitochondrial protein import signal. Only when a helix-destabilizing residue was substituted for a basic residue (R12-P) was function lost. In addition, when 
TABLE 4. Rules for a functional mitochondrial import signal

\begin{tabular}{lccc}
\hline $\begin{array}{c}\text { Potential rules: signal } \\
\text { functional if: }\end{array}$ & \multicolumn{2}{c}{ No. of mutants that conform to rules/total (\%) } \\
\cline { 2 - 4 } & $\begin{array}{c}\text { Single } \\
\text { mutants }\end{array}$ & $\begin{array}{c}\text { Double } \\
\text { mutants }\end{array}$ & $\begin{array}{c}\text { Total } \\
\text { mutants }\end{array}$ \\
\hline $\mathrm{HM}^{a}>5.5$ & $26 / 32(81)$ & $30 / 45(67)$ & $56 / 77(73)$ \\
$\mathrm{HM}>5.5$, no & $28 / 32(88)$ & $33 / 45(73)$ & $61 / 77(79)$ \\
$\quad \begin{array}{l}\text { acidic residues } \\
\mathrm{HM}>5.5, \text { no helix } \\
\text { breakers }\end{array}$ & $27 / 32(84)$ & $35 / 45(78)$ & $62 / 77(81)$ \\
$\begin{array}{l}\mathrm{HM}>5.5, \text { no } \\
\quad \begin{array}{l}\text { acidic residues or } \\
\text { helix breakers }\end{array}\end{array}$ & $29 / 32(91)$ & $38 / 45(84)$ & $67 / 77(87)$ \\
\hline
\end{tabular}

${ }^{a}$ HM, Hydrophobic moment.

another basic residue, lysine, was substituted for an arginine residue ( $\mathrm{R} 5-\mathrm{K})$, no significant change in the level of targeting was seen. Only at one position (leucine at position 6) did the wild-type residue appear to be critical for function. Each of three substitutions (L6-R, L6-P, and L6-Q) at this position resulted in a $\mathrm{Lct}^{-}$phenotype, decreasing $\beta$-subunit accumulation in mitochondria below $25 \%$ (Table 2 ). This amino acid residue may be important because it is a central component in the hydrophobic surface of the helix, and the substitutions characterized here may be detrimental because each of the mutations replaced this residue with either a hydrophilic or helix-destabilizing residue. However, an alternative possibility is that this residue provides a critical interaction with a component of the import machinery. If this were the case, this residue should be more highly constrained than would be predicted if it functioned solely as a component of the amphiphilic alpha helix. Additional experiments are required to distinguish between these possibilities.

Because of the observed correlation between the functional properties of most mutant signals and the amphiphilic alpha-helix model, we turned to a more quantitative measure of this trait. Hydrophobic moment values, which give a numerical value for the amphiphilicity of a defined portion of a protein $(7,8)$, were calculated for the $\mathrm{NH}_{2}$ terminus of the 77 single and double missense mutants by using constraints identical to those used by von Heijne (38). When these values were compared with the lactate growth phenotypes of each of the mutant isolates, a strong correlation was observed (Tables 2 and 4). For the single mutants, most targeting signals having a hydrophobic moment greater than 5.5 were capable of providing sufficient import to allow wild-type growth on lactate media, whereas signals having a value less than 5.5 were either $\operatorname{Lct}(\mathrm{Ts})$ or $\mathrm{Lct}^{-}$.

Besides the hydrophobic moment cutoff at 5.5, it was also observed that the introduction of acidic residues or helixdestabilizing residues (proline or glycine) almost always led to a respiration-defective [Lct(Ts) or $\mathrm{Lct}^{-}$] phenotype. When these three constraints were simultaneously compared with the lactate growth phenotype of the mutants, $91 \%$ of the single mutants and $87 \%$ of the combined single and double mutants conformed to these rules (Table 4). This high level of correlation demonstrates that these features represent important characteristics of a mitochondrial protein import signal.

\section{DISCUSSION}

The function of mitochondrial protein import signals has been proposed to depend on the ability of the signal to assume an amphiphilic alpha-helical structure. Evidence supporting this model comes from a statistical study that examined common characteristics of known mitochondrial protein targeting sequences (38) and from biophysical studies with synthetic peptides $(10,30,31)$. Although in many cases the results of these studies were consistent with the amphiphilic helix model, in vivo experiments that directly and systematically addressed the sequence and structural requirements of a mitochondrial protein import signal in a more physiological manner have largely been lacking. Most of the previous mutational studies of mitochondrial protein import signals have been limited to deletion and gene fusion studies that sought to define the smallest portion of a mitochondrial presequence that could still direct a measurable amount of protein import into mitochondria. Detailed analysis of the import properties of mutations generated by point mutagenesis has been used in only a few cases (16-18, 27 ), and a clear consensus of characteristics that are both necessary and sufficient to define a functional signal using such a genetic approach has not been obtained. In addition, It has recently been shown that some mutant precursors defective for mitochondrial import in vitro are still competent for import (to various extents) in vivo (20; Bedwell and Emr, unpublished results). Because of these findings, we feel that it is important to make a distinction between import defects based on in vitro and in vivo results.

The present study represents the most extensive systematic point mutational analysis of a minimal mitochondrial protein import signal. Analysis in the context of a minimal targeting sequence may be critical, since previously obtained results indicate that point mutations in the targeting signals of certain precursors can be phenotypically suppressed in the context of redundant targeting information $(2,27)$. A strong correlation was observed when the predicted structural and charge alterations caused by each mutation in the $\beta$-subunit minimal targeting sequence were compared with the lactate growth phenotype of the corresponding mutant. Together these observations have revealed a set of constraints that this signal must conform with to function properly. These include the following: (i) the hydrophobic moment for an alpha-helical structure near the $\mathrm{NH}_{2}$ terminus of the $\beta$-subunit precursor must be greater than 5.5 to direct a level of import sufficient to confer wild-type growth with lactate as the sole carbon source, (ii) at least two basic residues are necessary for efficient signal function, and (iii) acidic and, (iv) helix-breaking residues were found to actively interfere with the function of the targeting signal. When these rules were applied to our collection of 77 single and double point mutants, nearly $90 \%$ of the isolates conformed, indicating the accuracy of these simple rules in defining criteria important for a functional targeting signal (Table 4). However, it is important to note that the secondary structure predictions made for these mutant signals are based solely on a computer algorithm and remain to be confirmed experimentally. Other potentially important features that are implied but not tested directly in this study include the requirement that the $\mathrm{NH}_{2}$-terminal targeting signal must be accessible for recognition by the import machinery and the role that mature protein sequences may play in either facilitating import or in enhancing the activity of the $\mathrm{NH}_{2}$-terminal signal.

It should be noted, however, that these criteria are based on the effects of mutations within the context of the minimal targeting sequence encoded in residues 1 through 14 of the wild-type $\beta$-subunit precursor. Although this method is accurate in predicting the effects of mutations within this 
specific targeting sequence, caution should be used when applying these rules to predict whether an unknown sequence will function as a mitochondrial protein import signal. An analysis more specifically designed to discern mitochondrial protein targeting signals from nonmitochondrial proteins has been done by von Heijne (38). In that study, the amino acid sequences of 23 mitochondrial and 132 cytosolic proteins were used in a statistical analysis in which the $\mathrm{NH}_{2}$ termini of these proteins were compared with those of known surface-seeking peptides (such as calcitonin analogs). It was concluded that regions near the $\mathrm{NH}_{2}$ termini of mitochondrial precursors generally contain segments of high alpha-helical hydrophobic moment. By comparing the values of these proteins with those obtained for cytoplasmic proteins, a hydrophobic moment value of 7.3 (among other criteria) was assigned as the general cutoff for mitochondrial protein-targeting signals. Our calculations of hydrophobic moment values for the $\beta$-subunit minimal targeting sequence derivatives were done by using the same equation and scanning window size (18 residues) as those used by von Heijne (38), thereby allowing a direct comparison between values for the mutant $\beta$-subunit derivatives and natural mitochondrial protein targeting sequences. Although the WT(1-14) $\beta$-subunit value of 5.8 was well below the von Heijne cutoff point for a predicted mitochondrial protein import signal, it was near or above the values calculated for two natural mitochondrial import signals contained within the precursors of $\mathrm{Mn}$-superoxide dismutase and cytochrome oxidase subunit $\mathrm{Va}$. This is consistent with a single redundant domain of the $\beta$-subunit targeting sequence [represented here by the WT(1-14) minimal sequence] being within the limits of signal function but at a level allowing point mutations within this sequence to abolish most or all residual activity. The von Heijne cutoff point was made at the level of 7.3 to maintain a stringent discrimination between mitochondrial and cytosolic proteins. Perhaps if other, more subtle constraints (such as accessibility of the $\mathrm{NH}_{2}$ terminus) were taken into account, an equal degree of accuracy might be maintained at a lower level (such as 5.5 to 6.0). Alternatively, minor modifications of the algorithm used to calculate the hydrophobic moment value may increase the predictive accuracy. One such modification, in which the contribution of side chain flexibility was included in determining amphiphilicity, has recently been described (12).

It is often speculated that the surface-seeking properties of mitochondrial protein-targeting signals lead to the direct interaction of mitochondrial precursors with mitochondrial membrane(s). Although this may be true, it should not be overlooked that the same structures that give these sequences their membrane-seeking properties may also be critical for association with protein factors involved in the initial delivery of the precursor to the mitochondrial surface, in the interaction of the signal with a component (such as a receptor) on the mitochondrial surface, or in an interaction with component(s) of the translocation machinery. Based upon in vitro experiments, such factors appear to be essential for mitochondrial protein import $(26,29,39)$. Therefore, our isolation of point mutations within a mitochondrial protein targeting signal that may block import by abrogating one or more of these interactions could allow the selection of suppressor mutants that identify genes encoding these proteins. In preliminary experiments we have already obtained extragenic suppressors of certain $\mathrm{Lct}^{-} \beta$-subunit mutants (Bedwell, unpublished results). Further experiments are needed to determine whether these loci indeed encode gene products that participate directly in the process of mitochondrial protein import.

We have also isolated mutant signals that appear to target the $\beta$ subunit to mitochondria more efficiently than the WT(1-14) import signal. This result was both surprising and intriguing, since it could be assumed that the natural signal would be optimized to provide the most efficient possible rate of import. However, we have accumulated a considerable amount of evidence from both in vivo (2) and in vitro (Bedwell and Emr, unpublished results) experiments indicating that the reduction of the $\beta$-subunit signal to a single redundant domain greatly diminishes its import efficiency. It is not clear why certain proteins such as the $F_{1}$-ATPase $\beta$ subunit (2) and the alcohol dehydrogenase isozyme III (27) appear to have redundant targeting information, whereas other proteins, such as cytochrome oxidase subunit IV (19) and ornithine transcarbamylase (17) do not. Interestingly, it was recently reported that the region of ornithine transcarbamylase previously shown to be essential for in vitro mitochondrial import is not required for import in vivo (20). This result raises the possibility of redundancy in this mitochondrial protein import signal as well. Further studies will be needed to address the general importance of functional redundancy in mitochondrial protein-targeting signals.

Proteolytic processing of the $\beta$-subunit precursor by the matrix processing protease has been reported to occur between amino acid residues 19 and 20 of the precursor (35). This assignment was based upon the cleavage of an in vitro-synthesized $\beta$-subunit precursor by a soluble mitochondrial matrix extract. We have found that the $\beta$-subunit minimal targeting sequence constructs, which contained a deletion of residues 15 through 34 of the wild-type $\beta$-subunit precursor, were still processed by the matrix processing protease. This suggests that the recognition site for the matrix protease and the actual cleavage site may be physically separated in the precursor molecule. The minimal $\beta$-subunit constructs apparently still retain most or all of the recognition site, and this information is sufficient to provide efficient cleavage in most cases. This is unlike the cleavage site of secretory proteins, in which information directly adjacent to the processing site (between residues -5 and -1 with respect to the mature protein) is critical for cleavage of the signal sequence upon entry into the endoplasmic reticulum (37).

The nonselective aspect of the mutagenesis approach described here offers important advantages over classical methods that only allow the isolation of mutations leading to defective growth phenotypes. Phenotypically silent mutations can be as informative as mutations leading to growth defects, since they can define the limits of change that are allowed at a given amino acid position. In addition, when most of the candidates from a limited set of potential mutations are isolated, it greatly facilitates the analysis of double mutants by allowing the contributions of each mutation to be determined separately. By comparing the effect of a number of mutations both individually and in combination with a second mutation, a picture of the intimate interactions between the amino acid residues making up the targeting signal can be seen (Table 3 ). Clearly, mitochondrial proteintargeting signals do not function solely at the level of individual amino acids. Instead, interactions throughout the signal led to both positive and negative phenotypic effects on the function of the minimal targeting sequence in ways usually predicted by the rules outlined above. Ultimately, more extensive characterization of the functional and physical properties of these mutant targeting signals and a better 
understanding of the mutant signals that represent exceptions to the established rules may allow us to more completely understand the features that give mitochondrial protein targeting sequences their unique properties.

\section{ACKNOWLEDGMENTS}

We thank Suzanna Horvath for advice concerning the design of the mixed oligonucleotide, Jeff Schatz and Mike Douglas for providing antisera and monoclonal antibodies, and Keith Rosema for writing the computer program used to calculate the hydrophobic moment values. We also thank Bruce Horazdovsky for critically reading the manuscript.

This study was supported by Public Health Service grant GM32703 from the National Institues of Health. S.D.E. is a Presidential Young Investigator supported by National Science Foundation grant DCB-8451633. D.M.B. was supported by a Research Fellowship from the American Cancer Society. S.A.S. and G.D.J. were supported by a predoctoral training grant from the National Institutes of Health.

\section{LITERATURE CITED}

1. Baker, A., and G. Schatz. 1987. Sequences from a prokaryotic genome or the mouse dihydrofolate reductase gene can restore the import of a truncated precursor protein into yeast mitochondria. Proc. Natl. Acad. Sci. USA 84:3117-3121.

2. Bedwell, D. M., D. J. Klionsky, and S. D. Emr. 1987. The yeast $F_{1}$-ATPase $\beta$ subunit precursor contains functionally redundant mitochondrial protein import information. Mol. Cell. Biol. 7: 4038-4047.

3. Bradford, M. M. 1976. A rapid and sensitive method for the quantitation of microgram quantities of protein utilizing the principle of protein-dye binding. Anal. Biochem. 72:248-254

4. Casadaban, M. J., and S. N. Cohen.1980. Analysis of gene control signals by DNA fusion and cloning in Escherichia coli. J. Mol. Biol. 138:179-207.

5. Daum, G., P. C. Bohni, and G. Schatz. 1982. Import of proteins into mitochondria. Cytochrome $b_{2}$ and cytochrome $c$ peroxidase are located in the intermembrane space of yeast mitochondria. J. Biol. Chem. 257:13028-13033.

6. Douglas, M. G., M. T. McCammon, and A. Vassarotti. 1986. Targeting proteins into mitochondria. Microbiol. Rev. 50:166178.

7. Eisenberg, D. 1984. Three-dimensional structure of membrane and surface proteins. Annu. Rev. Biochem. 53:595-623.

8. Eisenberg, D., E. Schwarz, M. Komaromy, and R. Wall. 1984. Analysis of membrane and surface protein sequences with the hydrophobic moment plot. J. Mol. Biol. 179:125-142.

9. Emr, S. D., A. Vassarotti, J. Garrett, B. L. Geller, M. Takeda, and M. G. Douglas. 1986. The amino terminus of the yeast $F_{1}$-ATPase $\beta$-subunit precursor functions as a mitochondrial import signal. J. Cell. Biol. 102:523-533.

10. Epand, R. M., S.-W. Hui, C. Argan, L. L. Gillespie, and G. C. Shore. 1986. Structural analysis and amphiphilic properties of a chemically synthesized mitochondrial signal peptide. J. Biol. Chem. 261:10017-10020.

11. Gasser, S., G. Daum, and G. Schatz. 1982. Import of proteins into mitochondria. Energy-dependent uptake of precursors by isolated mitochondria. J. Biol. Chem. 257:13034-13041.

12. Gavel, Y., L. Nilsson, and Gunnar von Heijne. 1988. Mitochondrial targeting sequences: why 'non-amphiphilic' peptides may still be amphiphilic. FEBS Lett. 235:173-177.

13. Halvorson, H. O., and L. Ellias. 1958. The purification and properties of an $\alpha$-glucosidase of Saccharomyces italicus Y1225. Biochim. Biophys. Acta 30:28-40.

14. Hawlitschek, G., H. Schneider, B. Schmidt, M. Tropschug, F.-U. Hartl, and W. Neupert. 1988. Mitochondrial protein import: identification of processing peptidase and of PEP, a processing enhancing protein. Cell 53:795-806.

15. Hill, D. E., A. R. Oliphant, and K. Struhl. 1987. Mutagenesis with degenerate oligonucleotides: an efficient method for saturating a defined DNA region with base pair substitutions. Methods Enzymol. 155:558-568.
16. Horwich, A. L., F. Kalousek, and L. E. Rosenberg. 1985. Arginine in the leader peptide is required for both import and proteolytic cleavage of a mitochondrial precursor. Proc. Natl. Acad. Sci. USA 82:4930-4933.

17. Horwich, A. L., F. Kalousek, W. A. Fenton, R. A. Pollock, and L. E. Rosenberg. 1986. Targeting of pre-ornithine transcarbamylase to mitochondria: Definition of critical regions and residues in the leader peptide. Cell 44:451-459.

18. Horwich, A. L., F. Kalousek, W. A. Fenton, K. Furtak, R. A. Pollock, and L. E. Rosenberg. 1987. The ornithine transcarbamylase leader peptide directs mitochondrial import through both its midportion structure and net position charge. J. Cell. Biol. 105:669-677.

19. Hurt, E., D. S. Allison, U. Muller, and G. Schatz. 1987 Amino-terminal deletions in the presequence of an imported mitochondrial protein block the targeting function and proteolytic cleavage of the presequence at the carboxy terminus. $J$. Biol. Chem. 262:1420-1424.

20. Isaya, G., W. A. Fenton, J. P. Hendrick, K. Furtak, F. Kalousek, and L. E. Rosenberg. 1988. Mitochondrial import and processing of mutant human ornithine transcarbamylase precursors in cultured cells. Mol. Cell. Biol. 8:5150-5158.

21. Ito, H., Y. Fukuda, K. Murata, and A. Kimura. 1983. Transformation of intact yeast cells treated with alkali cations. J. Bacteriol. 153:163-168.

22. Keng, T., E. Alani, and L. Guarente. 1986. The nine aminoterminal residues of $\delta$-aminolevulinate synthase direct $\beta$-galactosidase into the mitochondrial matrix. Mol. Cell. Biol. 6: 355-364.

23. Maccecchini, M.-L., Y. Rudin, G. Blobel, and G. Schatz. 1979. Import of proteins into mitochondria: precursor forms of the extarmitochondrially made $F_{1}$-ATPase subunits in yeast. Proc. Natl. Acad. Sci. USA 76:343-347.

24. Messing, J., and J. Vieira. 1982. A new pair of M13 vectors for selecting either DNA strand of double-digest restriction fragments. Gene 19:269-276.

25. Miller, J. 1972. Experiments in molecular genetics. Cold Spring Harbor Laboratory, Cold Spring Harbor, N.Y.

26. Ohta, S., and G. Schatz. 1984. A purified precursor polypeptide requires a cytosolic protein fraction for import into mitochondria. EMBO J. 3:651-657.

27. Pilgrim, D., and E. T. Young. 1987. Primary structure requirements for correct sorting of the yeast mitochondrial protein ADHIII to the yeast mitochondrial matrix space. Mol. Cell. Biol. 7:294-304.

28. Reid, G. A., and G. Schatz. 1982. Import of proteins into mitochondria. Extramitochondrial pools and post-translational import of mitochondrial protein precursors in vivo. J. Biol. Chem. 257:13062-13067.

29. Riezman, H., R. Hay, C. Witte, N. Nelson, and G. Schatz. 1983. Yeast mitochondrial outer membrane specificity binds cytoplasmically-synthesized precursors of mitochondrial proteins. EMBO J. 2:1113-1118.

30. Roise, D., S. J. Horvath, J. M. Tomich, J. H. Richards, and G. Schatz. 1986. A chemically synthesized pre-sequence of an imported mitochondrial protein can form an amphiphilic helix and perturb natural and artificial phospholipid bilayers. EMBO J. 5:1327-1334

31. Roise, D., F. Theiler, S. J. Horvath, J. M. Tomich, J. H. Richards, D. S. Allison, and G. Schatz. 1988. Amphilicity is essential for mitochondrial presequence function. EMBO J. 7:649-653.

32. Sanger, F., S. Nicklen, and A. R. Coulson. 1977. DNA sequencing with chain-terminating inhibitors. Proc. Natl. Acad. Sci. USA 74:5463-5467.

33. Schleyer, M., and W. Neupert. 1985. Transport of proteins into mitochondria: translocation intermediates spanning contact sites between outer and inner membranes. Cell 43:339-350.

34. Sherman, F., G. R. Fink, and L. W. Lawrence. 1979. Methods in yeast genetics: a laboratory manual. Cold Spring Harbor Laboratory, Cold Spring Harbor, N.Y.

35. Vassarotti, A., W.-J. Chen, C. Smagula, and M. G. Douglas. 1987. Sequences distal to the mitochondrial targeting sequences 
are necessary for the maturation of the $F_{1}$-ATPase $\beta$-subunit precursor in mitochondria. J. Biol. Chem. 262:411-418.

36. Vassarotti, A., R. Stroud, and M. Douglas. 1987. Independent mutations at the amino terminus of a protein act as surrogate signals for mitochondrial import. EMBO J. 6:705-711.

37. Von Heijne, G. 1983. Patterns of amino acids near signal- sequence cleavage sites. Eur. J. Biochem. 133:17-21.

38. Von Heijne, G. 1986. Mitochondrial targeting sequences may form amphiphilic helices. EMBO J. 5:1335-1342.

39. Zwizinski, C., M. Schleyer, and W. Neupert. 1984. Proteinaceous receptors for the import of mitochondrial precursor proteins. J. Biol. Chem. 259:7850-7856. 